\title{
Comparative Performance of Different Malaria Diagnostic Tools among Pregnant Cohorts in Onitsha Southeast Nigeria
}

\author{
Christian Chibuzo Uba', ${ }^{1 *}{ }^{-}$, Moses Nkechukwu Ikegbunam², Ifeoma Sandra Anagor ${ }^{3}$, \\ Lilian Chinyere Eleanya ${ }^{4}$, Eucharia Nkiruka Ezeumeh ${ }^{5}$, Stephen Nnaemeka Ezekwueche ${ }^{4}$, \\ Edith Chinenye Okechukwu ${ }^{1}$, Charles Okechukwu Esimone ${ }^{2}$
}

\author{
${ }^{1}$ Department of Microbiology, Paul University, Awka, Nigeria \\ ${ }^{2}$ Department of Pharmaceutical Microbiology and Biotechnology, Nnamdi Azikiwe University, Awka, Nigeria \\ ${ }^{3}$ Department of Medical Microbiology and Parasitology, Nnamdi Azikiwe University, Awka, Nigeria \\ ${ }^{4}$ Department of Microbiology, Chukwuemeka Odumegwu Ojukwu University, Uli Campus, Uli, Nigeria \\ ${ }^{5}$ Department of Medical Laboratory Science, Chukwuemeka Odumegwu Ojukwu University, Igbariam Campus, Igbariam, Nigeria \\ Email: *christian.uba@pauluniversity.edu.ng, *uba.ucc@gmail.com
}

How to cite this paper: Uba, C.C., Ikegbunam, M.N., Anagor, I.S., Eleanya, L.C., Ezeumeh, E.N., Ezekwueche, S.N., Okechukwu, E.C. and Esimone, C.O. (2021) Comparative Performance of Different Malaria Diagnostic Tools among Pregnant Cohorts in Onitsha Southeast Nigeria. Advances in Infectious Diseases, 11, 384-394. https://doi.org/10.4236/aid.2021.114035

Received: November 3, 2021

Accepted: December 7, 2021

Published: December 10, 2021

Copyright () 2021 by author(s) and Scientific Research Publishing Inc. This work is licensed under the Creative Commons Attribution International License (CC BY 4.0).

http://creativecommons.org/licenses/by/4.0/ (c) (i) Open Access

\begin{abstract}
Background of Study: The reliability of microscopic techniques has become questionable in most endemic regions in Africa leading to its decreased utilization and increased utilization of RDT kits and other laboratory-based methods. Objective: To evaluate the performance of Rapid Diagnostic Test (RDT) kits and nest Polymerase Chain Reaction (nPCR) methods in detecting malaria infections among pregnant women visiting private hospitals in Onitsha district area of Anambra State, South-Eastern Nigeria. Methods: A total of 100 blood samples of pregnant women submitted to medical laboratory units of private maternal hospitals for malaria diagnosis in Onitsha district area were randomly selected for this study. Diagnosis was through microscopy, RDT kit (SD Bioline Pf-only test) and nPCR. Results: Pregnant cohorts had 95, 90 and 12 positive samples confirmed through microscopy RDT and nPCR respectively. RDT had a sensitivity and specificity of $89.47 \%$ and $0 \%$ while nPCR recorded sensitivity and specificity of $12.63 \%$ and $100 \%$ respectively. RDT and $\mathrm{nPCR}$ have a positive predictive value (PPV) of $94.44 \%$ of $100 \%$ respectively. Conclusion: This study revealed that nPCR is more efficient and reliable when compared with RDT in the diagnosis of malaria infection, having recorded the highest value for positive predictive value (PPV) and specificity than the RDT among pregnant women.
\end{abstract}

\section{Keywords}

RDT, Microscopy, PCR, Sensitivity, Specificity, Pregnancy 


\section{Introduction}

Each year, millions of people are infected with malaria parasite, with an undesirable effect of over one million deaths [1]. Pregnant women as well as young children, and non-immune visitors to malaria-endemic areas are at the greatest risk of severe or fatal malaria infection [1].

In Nigeria and other sub-Saharan African countries, $P$. falciparum is the leading cause of malaria in pregnant women [2]. WHO estimated 219 million malaria cases and 435,000 malaria deaths in 87 countries in 2017 and Nigeria accounts for $25 \%$ of these cases [3]. Pregnant women, as well as children under five years, have been reported to bear the greater burden of the disease with above $70 \%$ of all malaria deaths [4]. In sub-Saharan Africa, research has shown that over 30 million pregnant women per year are at risk of malaria [5]. In areas of stable $P$. falciparum malaria transmission, where approximately 50 million pregnancies occur each year, women are semi-immune and often carry their infections with few or no symptoms [2]. Among pregnant women in Nigeria, it has been reported that $30 \%(60 / 200)$ of the recruited women for a study on the prevalence of asymptomatic Plasmodium falciparum has asymptomatic $P$. falciparum infection [6]. Susceptibility to malaria increases during pregnancy, making these women an important parasite reservoir in the community [7].

The World Health Organization has recommended confirmation of all suspected malaria cases using microscopy-based techniques or rapid diagnostic tests (RDT) [8]. Often in regions where malaria infections are endemic, there are always multiple etiologies of fever and headache one of the leading symptoms of malaria [9]. These may however lead to misdiagnoses and hence wrong treatment, especially in children and pregnant women with compromised immunity. Also, the biology and clinical presentations of Plasmodium falciparum in semiimmune women have been shown to interfere with diagnosis during pregnancy, rendering targeted interventions ineffective for control [10].

Proper diagnosis and treatment of Malaria throughout endemic regions have always been a challenge and undefeatable issues, leading to its continued prevalence and increasing drug resistance [9]. Some of these challenges arise from inadequate resources, inexperienced technical personnel, poor diagnostic standards, and the lack of confidence in the currently available diagnostics among clinicians.

Through policy changes and the introduction of malaria rapid diagnostic tests (RDTs), diagnostic testing has improved, although microscopy-based methods such as bright field microscopy with Giemsa-stained specimens remain the gold standard for malaria diagnosis [8]. Malaria RDTs are a useful tool in countries where malaria is endemic, to counter the shortfalls of other diagnostic approaches especially in field studies where microscopy is not feasible [11]. They also have been used to complement microscopy as they provide timely results especially in settings where microscopy experience is limited as is the case in most non-endemic countries [12]. The RDT technique is based on the immu- 
nochromatographic principles, which capture antigens of the parasite including pan-lactate dehydrogenase (pLDH) or histidine-rich protein-2 (HRP-2) [13]. The detection threshold for RDTs is more than adequate for diagnosis of clinical cases. It becomes questionable when used among asymptomatic individuals [14].

Currently, polymerase chain reaction (PCR) assay has been employed in malaria diagnosis primarily for research purposes or for speciation in most laboratories. This method involves detecting circulating malaria parasites in blood or other bodily fluid by detecting parasite DNA through amplification of ribosomal RNA genes [15]. It is a highly specialized technique requiring expensive equipment and very elaborate settings. The polymerase chain reaction (PCR) allows the specific amplification of a selected region of the malarial genome [16]. This technique is highly specific and sensitive ( $1-5$ parasite $/ \mathrm{ml}$ of blood) and permits genotyping [17] [18].

Over-prescription and under-prescription of antimalarials are basically attributed to misdiagnosis of the malaria infection and hence efficient diagnosis of malaria parasite is very vital for treatment of malaria infection especially among pregnant women who are at greatest risk of severe or fatal malaria infection. Over the years, no data has been able to show and compare the performance and efficiency of RDT and PCR diagnostic tools among pregnant women in southeastern Nigeria who are important parasite reservoirs in the community. Therefore, in the current study, we evaluated the performance of the RDT and PCR methods in comparison to Giemsa stained microscopy as gold standard in pregnant women visiting private hospitals in Anambra state, southeastern Nigeria.

\section{Materials and Methods}

\subsection{Study Area}

This study was conducted at some selected maternity hospitals (Kanayo hospital and Gozie hospital) in Onitsha district area in Anambra state south Eastern Nigeria.

\subsection{Ethical Clearance}

Ethical Committee of Anambra State University Teaching Hospital, Amaku, Anambra State, Nigeria approved the study Protocol. Written informed consent was obtained from the participants

\subsection{Study Design}

Pregnant women suspected of having malaria based on the physician's tentative diagnosis were included in this study, while asymptomatic cohorts were excluded. One hundred (100) blood samples (determined using Cochran's equation formula) of symptomatic pregnant women submitted to the medical laboratory unit of the selected hospitals between October 2017 and December 2017 for malaria diagnosis were randomly selected for this study. Dried blood spot 
was made from pregnant women samples on a properly labelled filter paper (3 MM Whatman), and kept in a dry clean container with desiccant for a minimum of three hours to dry. The dried filter paper blood samples were stored at room temperature until when needed for further analyses.

\subsection{Microscopy}

This was done using standard procedures as proposed by WHO [19]. Thick and thin films were made from the blood samples in EDTA bottles; the films were stained with $10 \%$ Geimsa stain ( $\mathrm{pH}$ 7.2) for 10 minutes and examined under the microscope using $\times 1000$ magnification. Positive findings were graded on the thick smear using the "plus" system scale as previously described [20].

\subsection{Rapid Diagnostic Test}

The blood samples from all the symptomatic patients were tested using the SD Bioline (CAT No: 05FK90, Standard Diagnostics Inc., Korea, for Pf-only test). The test was performed following manufacturer's instructions. Negative results and Positive results as indicated by the presence of a single line, and double bands respectively in the strip were recorded.

\subsection{DNA Extraction}

DNA was extracted from the dried blood spots on filter paper using the QIAamp DNA Mini Kit (Qiagen, Hilden, Germany) following the manufacturer's protocol for DNA extraction in dried blood spot and stored in $-20^{\circ} \mathrm{C}$ until further use.

\subsection{Detection and Identification of Plasmodium Species}

Detection of plasmodium parasites was through a nested PCR, as previously described by [21] with slight modifications. For the Plasmodium genus detection a first PCR was done with specific primers rPLU1 and rPLU5 targeting 18S rRNA genes of $P$. falciparum, $P$. vivax, $P$. malariae, and $P$. ovale, followed by species specific nested PCR with rFAL1/rFAL2 primers.

The PCR reaction mix consists of $12.5 \mu \mathrm{l}$ of One Taq Quick-Load 2X Master Mix with Standard Buffer (New England Biolabs Inc.); $0.4 \mu \mathrm{l}$ each of forward and reverse primers; (forward primer: rPLU1:

TCAAAGATTAAGCCATGCAAGTGA; reverse primer: rPLU5:

CCTTGTTGTTGCCTTAACTTC) $6.7 \mu \mathrm{l}$ of Nuclease free water and $5 \mu \mathrm{l}$ of DNA template in a $25 \mu \mathrm{l}$ reaction volume. Amplification conditions for the first PCR step was as follows: Initial denaturation temperature of $95^{\circ} \mathrm{C}$ for 5 minutes, 25 cycles of denaturation at $94^{\circ} \mathrm{C}$ for 1 minute, primer annealing at $58^{\circ} \mathrm{C}$ for 1 minute and strand extension at $72^{\circ} \mathrm{C}$ for 5 minutes, using an Eppendorf nexus gradient Mastercycler (Germany).

For the nested, the PCR cocktail consists of $12.5 \mu \mathrm{l}$ of One Taq Quick-Load 2X Master Mix with Standard Buffer (New England Biolabs Inc.); $0.4 \mu \mathrm{l}$ each of 
forward and reverse primers; (forward primer RFAL1:

TTAAACTGGTTTGGGAAAACCAAAATATATT; reverse primer RFAL2: ACACAATGAACTCAATCATGACTACCCGTC), $9.7 \mu \mathrm{l}$ of Nuclease free water and $2 \mu \mathrm{l}$ of the PCR product of the first step in a $25 \mu \mathrm{l}$ reaction volume. Same amplification conditions were used for the 2nd step with 30 cycles for denaturation.

Each amplification run included one negative control (a negative control of DNA extract) and one positive control (Laboratory adapted 3D7 for P. falciparum). For nested-PCR reactions, an additional negative control was added, consisting of $2 \mu \mathrm{l}$ of the negative control reaction of the first run of PCR. The amplified products were visualized in $2 \%$ agarose gels stained with ethidium bromide.

\subsection{Data Analysis}

The sensitivity, specificity, and predictive values of each of the two test methods were calculated by comparing to Microscopy as the standard. The sensitivity, specificity, and predictive values of, rapid diagnostic test by SD Bioline and nested polymerase chain reaction (nPCR) results were then calculated using the formulas described by [22].

$$
\begin{gathered}
\text { Sensitivity }=[a /(a+c)] * 100 \\
\text { Specificity }=[d /(b+d)] * 100 \\
\operatorname{PPV}=[a /(a+b)] * 100
\end{gathered}
$$

where, $a=$ true positive, $b=$ false positive, $c=$ false negative, and $d=$ True negative.

All statistical analysis was performed using Microsoft Excel 2016 version.

\section{Results}

\subsection{Comparative Effect of Malaria Diagnostic Tools on Prevalence of Malaria}

A total number of one hundred samples blood samples submitted to the medical laboratory units were randomly selected for the comparative study. Microscopy recorded a total of 95 positive malaria cases and 5 negative cases. RDT revealed a total of 90 positive malaria cases and 10 negative results while PCR confirmed only 12 samples positive for malaria (Table 1). Statistical difference in prevalence rates was observed across the different diagnostic tools.

\subsection{Comparative Performance of Malaria Diagnostic Tools}

Of the ninety (90) samples declared to be positive for $P$. falciparum in peripheral blood of pregnant women by RDT, eighty-five (85) (94.4\%) samples received a positive diagnosis on the basis of peripheral blood microscopy, while five (5) (5.6\%) samples were confirmed negative by microscopy (Table 2). However, all the ten (10) samples that were declared negative by RDT received a positive diagnostic outcome by microscopy.

Out of the ninety-five (95) samples confirmed to be positive for $P$. falciparum 
in peripheral blood of pregnant women by microscopy, only twelve (12) samples received a positive diagnosis when subjected to $\mathrm{NPCR}$, while parasite DNA could not be detected in eighty-three (83) of the samples confirmed to be positive by microscopy.

Compared with microscopy as the gold standard, RDT was more sensitive (89.47\%) in detecting malaria cases than nPCR that showed a very low sensitivity (12.63\%) (Table 3). However, nPCR was more specific in detecting the presence of malaria parasite than $\mathrm{RDT}$ with a specificity value of $100 \%$ and $0 \%$ respectively. Also, nPCR showed a higher positive predictive value (PPV) (100\%) compared to RDT (94.44\%).

\section{Discussion}

Microscopic examination of Giemsa-stained thick and thin blood smears has been the diagnostic method of choice for Plasmodium species identification in epidemiologic studies and medical diagnosis [23]. This is based on the fact that

Table 1. Prevalence of malaria with respect to diagnostic tool.

\begin{tabular}{cccc}
\hline & \multicolumn{3}{c}{ Result } \\
\hline Tool $\mathrm{n}=100$ & Positive (\%) & Negative $(\%)$ & $\chi^{2},($ P-value $)$ \\
\hline Microscopy & $95(95)$ & $5(5)$ \\
RDT & $90(90)$ & $10(10)$ & $192.174,\left(\mathrm{P}<0.001^{\star}\right)$ \\
PCR & $12(12)$ & $88(88)$ & \\
\hline
\end{tabular}

$\chi^{2}=$ Pearson Chi-Square; ${ }^{\star}=$ P-value is significant at the 0.05 level.

Table 2. Malaria diagnosis by RDT and nPCRVersus detection of parasite through microscopy among study cohorts.

\begin{tabular}{ccccc}
\hline \multirow{2}{*}{ Tools } & & \multicolumn{2}{c}{ Microscopy (Gold Standard) } \\
\cline { 3 - 5 } & & Positive & Negative & Total \\
\hline \multirow{3}{*}{ RDT } & Positive & $85(94.4 \%)$ & $5(5.6 \%)$ & 90 \\
& Negative & $10(100 \%)$ & $0(0 \%)$ & 10 \\
& Total & 95 & 5 & 100 \\
\hline \multirow{2}{*}{ nPCR } & Positive & $12(100 \%)$ & $0(0 \%)$ & 12 \\
& Negative & $83(94.3 \%)$ & $5(5.7 \%)$ & 88 \\
& Total & 95 & 5 & 100 \\
\hline
\end{tabular}

Table 3. Comparative performance of RDT and PCR against Microscopy (Gold Standard) in malaria diagnosis among pregnant women.

\begin{tabular}{ccc}
\hline Performance & RDT & nPCR \\
\hline Sensitivity (\%) & 89.47 & 12.63 \\
Specificity (\%) & 0 & 100 \\
PPV (\%) & 94.44 & 100
\end{tabular}


the method is simple, and does not require highly equipped facilities, and in most cases enables differentiation among the four Plasmodium species causing malaria in human when performed by a trained medical laboratory technician. But yet there are existing questionable reliability of microscopic techniques arising from technicalities of the microscopist including the staining quality of the thick and thin smear, inability to differentiate between stained parasite cells and artifacts and or modification to parasite cells by drug treatment. All of these gave rise to the use of other tools like RDT and $\mathrm{nPCR}$ in detecting malaria parasites in blood samples of those with symptoms of malaria. The present study has shown the sensitivity and specificity of RDT and nPCR in whole blood samples from pregnant women while considering microscopy as the reference standard.

The Sensitivity of RDT as observed in this study is higher than 55\% and 77.7\% sensitivity reported by [20] in Awka, Anambra State South Eastern Nigeria but lower than $93.29 \%$ sensitivity reported by [24] in Adamawa state in Northern Nigeria although they were not reported for samples from pregnant women. However, Nnanna and colleagues had reported a higher sensitivity and specificity value of $100 \%$ and $96.1 \%$ respectively for RDT in a study of blood samples of 322 patients who attended four hospitals in Awka, Anambra state and were examined for malaria parasite infection [25]. The difference in sensitivity and specificity of RDT in this study compared with other reports could be associated with the difference in geographical zones, environmental conditions as well as storage practice in study locations. Study locations with extreme environmental conditions such as high temperature and humidity usually have a record of poor storage practices. This can eventually affect the performance of the RDT resulting to lower sensitivity and specificity [26]. Other scholars have argued that the presence of blocking antibodies may also contribute to reduced sensitivity of HRP2-detection tests [27]. The specificity of $0 \%$ recorded for RDT among the study cohorts indicate that there are no true negative cases [22]

False-positive results in RDT as observed in this study could be linked to the persistence of HRP2 antigen in patient blood weeks after successful treatment [28] especially in patients with high rheumatoid factor [29]. Furthermore, plasmodia gametocyte which persists despite clearance of asexual forms of parasite can also produce HRP2 and pLDH (Plasmodium lactate dehydrogenase) and thus could lead to a false positive [30].

False-negative results of RDT can occur due to presence of excess anti-HRP2 antibodies (prozone phenomenon) in humans [29] as well as recent reports of $P$. falciparum histidine-rich protein $2 / 3$ gene deletions [8]. Among pregnant women, false-negative RDT could be attributed to low parasite densities usually reported for pregnant women due to sequestered parasite biomass often limited to placental intervillous spaces (a theory which suggests that $1 \mathrm{ml}$ of blood from pregnant women will contain fewer parasites and parasite antigen compared with 1 $\mathrm{ml}$ of blood collected from a child or non-pregnant people [31].

The introduction of PCR based methods has been shown to be a powerful tool 
for malaria diagnosis over time. This study, however, recorded a lower sensitivity but proved to be more specific for the detection of malaria parasites. The lower sensitivities observed in this study do not agree with the reports of other studies elsewhere that have shown that nested PCR, is more sensitive $(>80.4 \%)$ for detection of malaria parasites than other diagnostic tools [32] [33]. Over the decade, PCR based methods have shown varying sensitivities and specificities in Nigeria although with limited data available for the southeastern part of the country, especially in pregnant women. Sensitivity and specificity of $29.4 \%$ and $100 \%$ was obtained in a study in Ile-Ife [34] while sensitivity and specificity of $53.8 \%$ and $100 \%$ were reported in Lagos [35]. PCR technique, although more laborious and expensive than microscopy, have shown better diagnostic accura$c y$ and are highly useful for the detection of $P$. falciparum and other malaria species in asymptomatic and low parasitaemia cases. In the present study, Giemsa stained slides (thick films) for microscopy failed to detect the parasite in some positive samples by nPCR. False negatives as proposed by other scholars could pose a big public health problem because the patient would miss correct diagnosis and hence treatment, thereby not acting in accordance with WHO's recommendation of rapid and correct diagnosis, followed with treatment of confirmed presence of malaria parasite. The implications of this kind of misdiagnosis could lead to decline in health, increased transmission, and may be death.

Furthermore, cases of false-positive results for the diagnostic tools evaluated in this study pose a serious challenge to the fight against malaria, as this kind of misdiagnosis would result to wrong prescription and treatment in other words drug abuse which could lead to resistance to antimalarial drug.

Finally, the sensitivity and specificity of RDT and nPCR are dependent on the results of the microscopy (reference standard) with the potentials of recording false positive. False-positive results in microscopy can result from inadequately trained staff that report artifacts as positive result, while false-negative results in microscopy may be due to inadequately trained staff that cannot recognize the parasite or stain the test specimen properly. Since accurate diagnostic methods especially microscopy is the basis for adequate disease control efforts should be made to extend microscopy training to health facilities located in rural settings.

The limitations of this study include the absence of quantitative assessment of parasite density using microscopy and PCR methods, as well as a small sample size due to resource constraints. However, these constraints will be taken into account in future studies in order to incorporate the parasite level into the comparative analytic discourse.

\section{Conclusion}

This study established that nPCR is more efficient and reliable in the diagnosis of malaria parasites among pregnant women having recorded the highest value for PPV and Specificity than the RDT. However, RDTs can continue to serve as a useful tool in the early diagnosis of malaria in infected persons as it competes 
with nPCR favorably in availability and cost especially in developing regions including Nigeria. We encourage the continued use of microscopy data from a WHO-certified microscopist as a reference standard for prevalence and comparative studies while nPCR should be used to confirm negative results from microscopy and RDT if used.

\section{Conflicts of Interest}

The authors declare no conflicts of interest regarding the publication of this paper.

\section{References}

[1] Rogerson, S.J., Desai, M., Mayor, A., Sicuri, E., Taylor, S.M. and van Eijk, A.M. (2018) Burden, Pathology, and Costs of Malaria in Pregnancy: New Developments for an Old Problem. The Lancet Infectious Diseases, 18, e107-e118. https://doi.org/10.1016/S1473-3099(18)30066-5

[2] Yimam, Y., Nateghpour, M., Mohebali, M. and Abbaszadeh Afshar, M.J. (2021) A Systematic Review and Meta-Analysis of Asymptomatic Malaria Infection in Pregnant Women in Sub-Saharan Africa: A Challenge for Malaria Elimination Efforts. PLoS ONE, 16, Article ID: e0248245. https://doi.org/10.1371/journal.pone.0248245

[3] WHO (2018).Global Malaria Programme. https://www.who.int/teams/global-malaria-programme/reports/world-malaria-repo $\underline{\mathrm{rt}-2018}$

[4] Anto, F., Agongo, I.H., Asoala, V., Awini, E. and Oduro, A.R. (2019) Intermittent Preventive Treatment of Malaria in Pregnancy: Assessment of the Sulfadoxine-Pyrimethamine Three-Dose Policy on Birth Outcomes in Rural Northern Ghana. Journal of Tropical Medicine, 2019, Article ID: 6712685. https://doi.org/10.1155/2019/6712685

[5] Gontie, G.B., Wolde, H.F. and Baraki, A.G. (2020) Prevalence and Associated Factors of Malaria among Pregnant Women in Sherkole District, Benishangul Gumuz Regional State, West Ethiopia. BMC Infectious Diseases, 20, Article No. 573. https://doi.org/10.1186/s12879-020-05289-9

[6] Ojurongbe, O., Nguetse, C.N., Fayemiwo, S.A., Falade, C.O., Ojurongbe, T.A., Thomas, B.N., et al. (2018) High Prevalence of Dihydrofolate Reductase Gene Mutations in Plasmodium falciparum Parasites among Pregnant Women in Nigeria after Reported Use of Sulfadoxine-Pyrimethamine. Pathogens and Global Health, 112, 86-92. https://doi.org/10.1080/20477724.2017.1422615

[7] Healy, S.A., Fried, M., Richie, T., Bok, K., Little, M., August, A., Riley, L., Swamy, G.K., Wylie, B.J., Menendez, C. and Muehlenbachs, A. (2019) Malaria Vaccine Trials in Pregnant Women: An Imperative without Precedent. Vaccine, 37, 763-770. https://doi.org/10.1016/j.vaccine.2018.12.025

[8] World Health Organization (2017) In Kenya, the Path to Elimination of Malaria Is Lined with Good Preventions. http://www.who.int/features/2017/vector-control-kenya/en/

[9] Parsel, S.M., Gustafson, S.A., Friedlander, E., Shnyra, A.A., Adegbulu, A.J., Liu, Y., et al. (2017) Malaria Over-Diagnosis in Cameroon: Diagnostic Accuracy of Fluorescence and Staining Technologies (FAST) Malaria Stain and LED Microscopy versus Giemsa and Bright Field Microscopy Validated by Polymerase Chain Reaction. Infectious Diseases of Poverty, 6, Article No. 32. 
https://doi.org/10.1186/s40249-017-0251-0

[10] Wirth, D.F. and Alonso, P.L. (2017) Biology in the Era of Eradication. John Inglis, New York

[11] Boyce, M.R. and O'Meara W.P. (2017) Use of Malaria RDTs in Various Health Contexts across Sub-Saharan Africa: A Systematic Review. BMC Public Health, 17, Article No. 470. https://doi.org/10.1186/s12889-017-4398-1

[12] Aju-Ameh, C.O. (2019) Time to Track All Malaria Parasites in Nigeria. Science World Journal, 14, 39-41.

[13] Martín-Díaz, A., Rubio, J.M., Herrero-Martínez, J.M., Lizasoain, M., Ruiz-Giardin, J.M., Jaqueti, J., et al. (2018) Study of the Diagnostic Accuracy of Microbiological Techniques in the Diagnosis of Malaria in the Immigrant Population in Madrid. Malaria Journal, 17, Article No. 314. https://doi.org/10.1186/s12936-018-2459-2

[14] Mathison, B.A. and Pritt, B.S. (2017) Update on Malaria Diagnostics and Test Utilization. Journal of Clinical Microbiology, 55, 2009-2017. https://doi.org/10.1128/JCM.02562-16

[15] Kandie, R., Ochola, R. and Njaanake, K. (2018) Evaluation of Fluorescent in-Situ Hybridization Technique for Diagnosis of Malaria in Ahero Sub-County Hospital, Kenya. BMC Infectious Diseases, 18, Article No. 22. https://doi.org/10.1186/s12879-017-2875-x

[16] Picot, S., Cucherat, M. and Bienvenu, A.L. (2020) Systematic Review and Meta-Analysis of Diagnostic Accuracy of Loop-Mediated Isothermal Amplification (LAMP) Methods Compared with Microscopy, Polymerase Chain Reaction and Rapid Diagnostic Tests for Malaria Diagnosis. International Journal of Infectious Diseases, 98, 408-419. https://doi.org/10.1016/j.ijid.2020.07.009

[17] de la Serna, E., Arias-Alpízar, K., Borgheti-Cardoso, L.N., Sanchez-Cano, A., Sulleiro, E., Zarzuela, F., et al. (2021) Detection of Plasmodium falciparum Malaria in $1 \mathrm{~h}$ Using a Simplified Enzyme-Linked Immunosorbent Assay. Analytica Chimica Acta, 1152, Article ID: 338254. https://doi.org/10.1016/j.aca.2021.338254

[18] van Bergen, K., Stuitje, T., Akkers, R., Vermeer, E., Castel, R. and Mank, T. (2021) Evaluation of a Novel Real-Time PCR Assay for the Detection, Identification and Quantification of Plasmodium Species Causing Malaria in Humans. Malaria Journal, 20, Article No. 314. https://doi.org/10.1186/s12936-021-03842-8

[19] Research Malaria Microscopy Standards Working Group (2015) Microscopy for the Detection, Identification and Quantification of Malaria Parasites on Stained Thick and Thin Films. World Health Organization, Geneva.

[20] Anagu, O.L., Ikegbunam, M.N., Unachukwu, C.K., Ogwaluonye Uchenna, C. and Esimone, C.O. (2015) Comparison of Microscopic Determination and Rapid Diagnostic Tests (RDTs) in the Detection of Plasmodium Infection. Advances in Microbiology, 5, 604-609. https://doi.org/10.4236/aim.2015.58063

[21] Siwal, N., Singh, U.S., Dash, M., Kar, S., Rani, S., Rawal, C., et al. (2018) Malaria Diagnosis by PCR Revealed Differential Distribution of Mono and Mixed Species Infections by Plasmodium falciparum and P. vivax in India. PLOS ONE, 13, Article ID: e0193046. https://doi.org/10.1371/journal.pone.0193046

[22] Trevethan R. (2017) Sensitivity, Specificity, and Predictive Values: Foundations, Pliabilities, and Pitfalls in Research and Practice. Frontiers in Public Health, 5, Article No. 307. https://doi.org/10.3389/fpubh.2017.00307

[23] Gitta, B. and Kilian, N. (2020) Diagnosis of Malaria Parasites Plasmodium spp. in Endemic Areas: Current Strategies for an Ancient Disease. BioEssays, 42, Article ID: 1900138. https://doi.org/10.1002/bies.201900138 
[24] Bashir, M., Sunday, E., Mohammed, B., Rufa, I., Isa, H., Sambo, K.H. and Adamu, M.K. (2019) Evaluation of the Efficacy of Rapid Diagnostic Tests Compared to Microscopy in the Diagnosis of Malaria Infection. International Journal of Mosquito Research, 6, 37-41.

[25] Nnanna, A.D., Chidiebere, N.S., Benedicta, A.O., Shedrack, E.O. and Kosisochukwu, U.I. (2018) Evaluation of Malaria Prevalence Using Microscopic Examination and Histidine Rich Protein-2 Rapid Diagnostic Test in Anambra State, Nigeria. AASCIT Journal of Health, 5, 16-20.

[26] Mfuh, K.O., Achonduh-Atijegbe, O.A., Bekindaka, O.N., Esemu, L.F., Mbakop, C.D., Gandhi, K., Leke, R.G., Taylor, D.W. and Nerurkar, V.R. (2019) A Comparison of Thick-Film Microscopy, Rapid Diagnostic Test, and Polymerase Chain Reaction for Accurate Diagnosis of Plasmodium falciparum Malaria. Malaria journal, 18, Article No. 73. https://doi.org/10.1186/s12936-019-2711-4

[27] Markwalter, C.F., Mudenda, L., Leelawong, M., Kimmel, D.W., Nourani, A., Mbambara, S., Thuma, P.E. and Wright, D.W. (2018) Evidence for Histidine-Rich Protein 2 Immune Complex Formation in Symptomatic Patients in Southern Zambia. Malaria Journal, 17, Article No. 256. https://doi.org/10.1186/s12936-018-2400-8

[28] Poti, K.E., Balaban, A.E., Pal, P., Kobayashi, T., Goldberg, D.E., Sinnis, P. and Sullivan, D.J. (2019) In Vivo Compartmental Kinetics of Plasmodium falciparum Histidine-Rich Protein II in the Blood of Humans and in BALB/c Mice Infected with a Transgenic Plasmodium berghei Parasite Expressing Histidine-Rich Protein II. Malaria Journal, 18, Article No. 78. https://doi.org/10.1186/s12936-019-2712-3

[29] Poti, K.E., Sullivan, D.J., Dondorp, A.M. and Woodrow, C.J. (2020) HRP2: Transforming Malaria Diagnosis, but with Caveats. Trends in Parasitology, 36, 112-126. https://doi.org/10.1016/j.pt.2019.12.004

[30] Arora, B.K. and Arora, S. (2020) Chapter-1 Malaria Diagnostics: Tools and Trends. Chief Editor Dr. Durgadas Govind Naik, 105, 1.

[31] Dayanand, K.K., Achur, R.N. and Gowda, D.C. (2018) Epidemiology, Drug Resistance, and Pathophysiology of Plasmodium vivax Malaria. Journal of Vector Borne Diseases, 55, 1-8. https://doi.org/10.4103/0972-9062.234620

[32] Mahende, C., Ngasala, B., Lusingu, J., Yong, T.S., Lushino, P., Lemnge, M., Mmbando, B. and Premji, Z. (2016) Performance of Rapid Diagnostic Test, Blood-Film Microscopy and PCR for the Diagnosis of Malaria Infection among Febrile Children from Korogwe District, Tanzania. Malaria Journal, 15, Article No. 391. https://doi.org/10.1186/s12936-016-1450-z

[33] Adedoja, A., Babatunde, S.K., Tijani, B.D., Akanbi, A.A. and Ojurongbe, O. (2019) Usefulness of Polymerase Chain Reaction in the Diagnosis of Asymptomatic Malaria among School Age Children in Ilorin, Nigeria. Pan African Journal of Life Sciences, 1, 39-45. https://doi.org/10.36108/pajols/8102/10(0170)

[34] Ogbolu, D.O., Alli, O.A.T., Nassar, A.S. and Ajagbe, O.O. (2012) Evaluation of Usefulness of Polymerase Chain Reaction in the Diagnosis of Malaria in Nigeria. African Journal of Clinical and Experimental Microbiology, 13, 126-134. https://doi.org/10.4314/ajcem.v13i3.1

[35] Kanyi, O.I., Ajayi, M.B., Ezeugwu, S.M.C., Afocha, E.E. and Iwalokun, E. (2016) Comparison of Rapid Diagnostic Test (RDT), Polymerase Chain Reaction (PCR) And Microscopy Methods in The Diagnosis of Malaria among Airport Workers In Lagos. Nigerian Journal of Science and Environment, 13, 18-24. 